pregnancy and infant outcomes and may also increase the risk of HIV mother-to-child-transmission. Additionally, these infections may be vertically transmitted to neonates, resulting in colonisation, conjunctivitis, and pneumonia. There is a lack of data on the neonatal incidence of CT, NG and TV due to lack of systematic screening of mothers and neonates and reliance on STI syndromic management. We conducted an analysis to determine rates of vertical transmission of CT, NG and TV from HIV-infected women to their neonates.

Methods We tested HIV-infected pregnant women for CT, NG and TV on vulvo-vaginal swab specimens during their first clinic visit post-delivery. Neonates of all women who tested positive for an STI were also evaluated for these STIs using nasopharyngeal swab specimens collected the same day. Testing was done using the Xpert CT/NG and Xpert TV [Cepheid, Sunnyvale, CA].

Results Of the 54 women tested for CT, NG and TV postnatally, $27(50 \%)$ had at least one STI. Twenty three $(85.2 \%)$ of the 27 neonates born to women with STIs were evaluated for STI colonisation. Overall, 18 (78.3\%) neonates tested positive for at least one STI; 77.3\% (17/22) neonates born to mothers with CT infection, 50\% (2/4) with TV, and none with NG tested positive for those respective infections. STI colonisation rates also differed by birth weight with $100 \%$ of low birth weight, $77.8 \%(14 / 18)$ with normal birth weight, and 50\% (1/ 2) of high birth weight neonates testing positive for at least one of those infections. Rates of neonatal STI colonisation $(83.3 \%)$ were also high among the six infants delivered by Csection.

Conclusion Our analysis revealed high rates STIs among postpartum HIV-infected women, coupled with high rates of neonatal STI colonisation, particularly among low birth weight neonates.

\section{P3.142 EPIDEMIOLOGICAL AND CLINICAL PROFILE OF HIV PATIENTS OF A PUBLIC HEALTH UNIT FROM BELO HORIZONTE, MINAS GERAIS}

MI Maciel, SVF Carvalho, LM Gomes, JM Freire, CG Coelho. Municipal Health Department, Belo Horizonte, Brazil, Belo Horizonte - MG, Brazil

\subsection{6/sextrans-2017-053264.377}

Introduction More than 800000 individuals are HIV-infected in Brazil and a small increase of new infections (4\%) was observed in past years. Nowadays, more than half $(64 \%)$ of people living with HIV in Brazil are receiving antiretroviral treatment (ARVT). The epidemiological and clinical profiles of HIV patients in medium-sized brazilian cities are unknown, and it is necessary to study them to allow comparisons. The present study aims to identify the profile of HIV-infected individuals in a public health unit, from 2013 to 2016.

Methods A descriptive study was carried out in a public health care unit, which has specialised care for HIV-infected and sexually transmitted infections (STIs). The population of interest in this study were patients (319 patients) infected with HIV and under ARVT. Individuals whose registry was in duplicate $(n=1)$, whose ARVT started before $2013(n=8)$, and those who abandoned ARVT $(n=26)$ were excluded. Demographic data (age, sex and self-identified sexual identity) and information on the patients' clinical situation (HIV diagnosis year, ARVT start date, presence of undetectable viral load (VL) and time to undetectable VL) were obtained. Descriptive analysis of quantitative variables was performed using the Stata software, version 12.0 .

Results Among the 285 patients included in the study, most of them were male (89.44\%), aged 20-29 years (47.35\%) and self-identified their sexual identity as homosexual (69.01\%). Most of the diagnoses were performed in the year 2015 $(39.08 \%)$ and the majority of the patients had an undetectable VL (78.95\%). The mean time for VL to become and remain undetectable after starting ARVT was 7.49 (+6.74) months.

Conclusion The present study reflects the epidemiology of HIV infection in Brazil, with concentration of the disease in young adults and men who have sex with men. The virological response to ARVT is directly related to adherence to regular treatment and should be the target investment of health services specialised to people with HIV/AIDS.

\section{P3.143 SEROLOGICAL PREVALENCE OF ANTIBODIES TO HUMAN IMMUNODEFICIENCY VIRUS AMONG NEPALESE POPULATION}

${ }^{1}$ Manoj Kumar Sah, ${ }^{2}$ Prabhakar Verg Shah, ${ }^{3}$ Rupa Adhikari, ${ }^{4}$ Shyam Kumar Mishra, ${ }^{4}$ Keshab Parajuli. 'Kathmandu University, Kantipur Dental College Teaching Hospital and Research Centre, Kathmandu, Nepal; ${ }^{2} B$ and B Hospital, Kathmandu, Nepal; ${ }^{3}$ Namaste Nepal Medical Centre, Kathmandu, Nepal; ${ }^{4}$ Tribhuvan University Teaching Hospital, Kathmandu, Nepal

\subsection{6/sextrans-2017-053264.378}

Introduction HIV is one of the major public health problems in recent time. Because of limited work opportunities in Nepal, seasonal labour migration especially disadvantaged groups to overseas become common. Nepalese government is promoting overseas employment opportunities' for its citizens because of poor absorption of growing labour force in the country. This study was aimed to determine the serological prevalence of antibodies Human Immunodeficiency Virus (HIV) among the Nepalese population intended to depart overseas.

Methods This study was conducted at Namaste Nepal Medical Centre and Polyclinic, Kathmandu, Nepal. Twenty Nine Thousand Four Hundred Forty One healthy populations were included for this study over a period from January 2013 to December 2014. The venous blood was collected aseptically and subjected to screening for anti HIV 1/2 (HIV Tri Dot, Jay Mitra, India) and confirmed by third generation HIV $1 / 2$ ELISA (SD, Bio Lines, Korea) prior to overseas migration for labour employment. Data were analysed by using SPSS version 17.0 software.

Results The serological prevalence of antibodies to HIV $1 / 2$ were found to be $0.04 \% \quad(11 / 29,441)$. Among the HIV infected population $0.03 \%(9 / 29,441)$ was male while only $0.01 \%(2 / 29,441)$ was female. Majority of the HIV infected people with HIV 1/2 was found to be 20-30 years old and belonging from disadvantaged groups like Dalit, Janajati and Kshetri.

Conclusion The HIV seroprevalence showed decreasing trend, due to the cumulative effect of increasing awareness of HIV and better HIV testing and counselling (HTC) in Nepal. This study reflects that it would be alarming situation because young age population becomes more infected. Therefore, a continuous surveillance, advocacy and social mobilisation need to be considered for further improvements of transmission of HIV infection. 